\title{
Patrón de movimientos y ámbito hogareño invernal de aves de pastizal en el noroeste de México
}

\author{
Movement pattern and winter home range of grassland birds in northwest Mexico
}

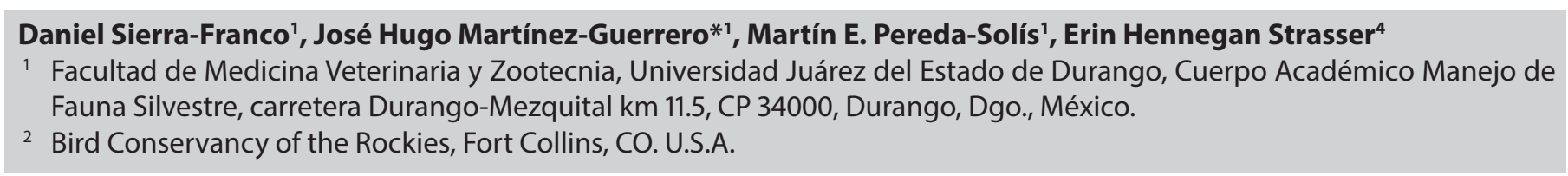

\section{RESUMEN}

La región de Cuchillas de la Zarca es considera como un área prioritaria para la conservación de pastizales reconocida por CONABIO la cual alberga una gran cantidad de aves migratorias durante el invierno. En este estudio calculamos el tamaño del ámbito hogareño, los patrones de movimiento y estructura de la vegetación de dos especies de pastizal el gorrión de Baird (Centronyx bairdii) y el gorrión chapulinero (Ammodramus savannarum). Durante el invierno 2013 - 2014 colocamos 33 transmisores; de los cuales se obtuvieron un total de 1440 registros de detección. El análisis de los datos espaciales permitió definir que la especie Centronyx bairdii $(n=20)$ recorre en promedio mayor distancia (203.13 $\mathrm{m} \pm$ DE 187.07) y ocupa mayor área (4.82 ha \pm 1.86 IC $95 \%$ ) que Ammodramus savannarum $(\mathrm{n}=13,134.31 \mathrm{~m} \pm \mathrm{DE} 106.3$ y 4.38 \pm 2.35 IC $95 \%$ ). El análisis de la vegetación permitió suponer que Centronyx bairdii prefiere sitios con mayor cobertura de pasto $(66.76 \pm 0.6)$ a diferencia de Ammodramus savannarum que prefirió sitios con pastizal más alto (36.06 \pm 0.6$)$. Las medidas geoespaciales y la estructura de la vegetación calculadas en este estudio pueden utilizarse como valores de referencia de ambas especies y pueden aportar conocimiento y entendimiento de su estadía no reproductiva.

Palabras clave: Desierto Chihuahuense, Telemetría, Vegetación, Centronyx bairdii, Ammodramus savannarum.

\section{ABSTRACT}

The Cuchillas de la Zarca region is considered as a priority area for the conservation of grasslands recognized by CONABIO which houses a large number of migratory birds during winter. In this study, we calculated the size of the home range, movement patterns and vegetation structure of two grassland species: the Baird's sparrow (Centronyx bairdii) and the grasshopper sparrow (Ammodramus savannarum). During the 2013-2014 winter we placed 33 transmitters; of which a total of 1440 detection records were obtained. The analysis of the spatial data allowed us to define that Centronyx bairdii $(\mathrm{n}=20)$ crosses in average a greater distance (203.13 $\mathrm{m} \pm$ DE 187.07) and occupies a greater area (4.82 ha \pm 1.86 IC $95 \%$ ) than Ammodramus savannarum ( $n=13,134.31$ $\mathrm{m} \pm$ SD 106.3 and $4.38 \pm 2.35$ IC $95 \%)$. The vegetation analysis allowed us to suppose that Centronyx bairdii prefers sites

*Autores para correspondencia: José Hugo Martínez-Guerrero Correo electrónico: che_hugo1@hotmail.com with greater grass cover $(66.76 \pm 0.6)$ unlike Ammodramus savannarum that preferred sites with higher grassland (36.06 \pm 0.6 ). The geospatial measurements and vegetation structure calculated in this study can be used as reference values of both species and can provide knowledge and understanding of their non-reproductive stay.

Keywords: Chihuahuan Desert, Telemetry, Vegetation, Centronyx bairdii, Ammodramus savannarum.

\section{INTRODUCCIÓN}

En Norteamérica el grupo de las aves de pastizal, es uno de los más vulnerables, ya que $75 \%$ de sus especies muestra una tendencia negativa en sus poblaciones ( $\mathrm{NABCl}$, 2016; Sauer et al., 2017. En particular, el gorrión de Baird (Centronyx bairdii) y el gorrión chapulín (Ammodramus savannarum) presentan tasas de disminución anual particularmente altas de -2.1 y $-2.5 \%$ respectivamente (Sauer et al., 2017).

Los factores que se han asociado con este patrón de disminución son la fragmentación y pérdida del hábitat debido al incremento de la frontera agrícola, el sobrepastoreo, la urbanización y la expansión de especies invasivas (Hoekstra et al., 2005), que afectan tanto las zonas de reproducción como las de invierno, principalmente en el Desierto Chihuahuense mexicano (Pool et al., 2014).

En ese sentido, la investigación acerca de la ecología invernal de estas y otras especies obligadas de pastizal apenas inicia y es escaza (Vickery y Herkert, 2001; Ruth et al., 2014), especialmente en los sitios de invernada del Desierto Chihuahuense mexicano (CEC, 2013), donde se han realizado algunos estudios para conocer algunos aspectos ecológicos importantes como abundancia y distribución (Panjabi et al., 2007) densidad y distribución invernal (Martínez-Guerrero et al., 2011), sobrevivencia invernal (Macias-Duarte y Panjabi, 2013), estructura de la vegetación y condiciones climáticas en las densidades de las aves (Macias-Duarte et al., 2018). Donde, la información respecto al uso de hábitat, ámbito hogareño y patrón de movimientos durante la temporada invernal es limitada con algunos trabajos realizados en Texas, E.U. y Chihuahua, México (Macias-Duarte y Panjabi, 2013; Gordon 2000a; Gordon, 2000b).Tanto Centronyx bairdii como Ammodramus savannarum se reconocen como aves focales de pastizal y debido a la fragmentación y degradación de su hábitat se encuentran en estado de preocupación notable 
(NACBI, 2016), de preocupación continental ( PIF, 2016), y como especies protegidas en Estados Unidos y Canadá.

Por lo anterior se planteó el objetivo de estimar los patrones de movimiento y ámbito hogareño de Centronyx bairdii (BAIS) y Ammodramus savannarum (GRSP), en el área prioritaria para la conservación de pastizales (APCP) de Cuchillas de la Zarca, en el Desierto Chihuahuense.

\section{MATERIALES Y MÉTODOS}

El estudio se llevó a cabo durante el periodo invernal 2013-2014 en el rancho Santa Teresa ubicado en el Municipio de Hidalgo al noroeste del Estado de Durango (figura 1) en tres áreas de pastizal: Las Yeguas (LAYE), Doña Wencha (DOWE) y La Laguna (LALA), dentro del APCP de Cuchillas de la Zarca.

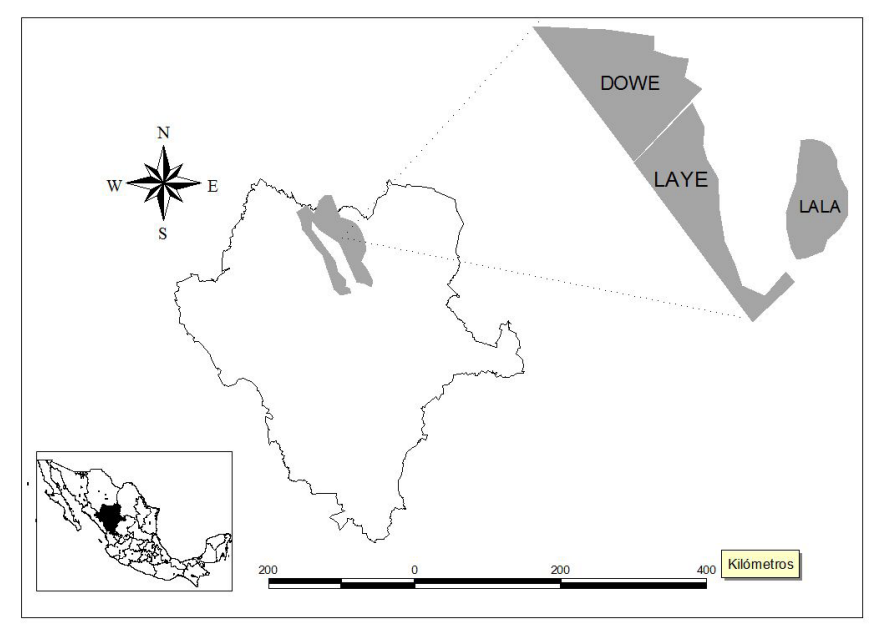

Figura 1. Área de estudio en el Rancho Santa Teresa, dentro del Área Prioritaria para la Conservación de Pastizales (APCP) Cuchillas de la Zarca, Durango, México.

Figure 1. Study area at Rancho Santa Teresa, within the Grasslands Priority Conservation Area (GPCA) Cuchillas de la Zarca, Durango, Mexico.

Para la captura de aves, se utilizaron 4 redes de niebla de 12 metros de largo y 2.60 metros de alto de polyester negro modelo KTX de Avian Research Supplies, AFO, de $36 \mathrm{~mm}$ de malla y la ayuda de ocho personas para usar el método de arreo de las aves hacia la red (Panjabi y Beyer ,2010) avalado con permiso de colecta de SEMARNAT SPGA-DGVS-13360-14.

Después de la captura a cada una de las aves se le colocó un anillo metálico talla 1 del USGS (United States Geological Survey), para su identificación y se registraron los datos de especie, largo de ala $(\mathrm{mm})$, largo cola $(\mathrm{mm})$ con una regla metálica para ala de $150 \mathrm{~mm}$ WING15ECO de AVINET', pico culmen $(\mathrm{mm})$, pico ancho $(\mathrm{mm})$, pico profundidad $(\mathrm{mm})$, con un vernier modelo SPI 150 mm de AVINET', categoría de grasa y edad en base a Pyle (1997) y peso (gr) con una báscula digital portátil modelo ACCULAB EC-211 con capacidad de $201 \mathrm{~g}$, con una precisión de $0.1 \mathrm{~g}$ de SARTORIUS:

Posteriormente se colocó un transmisor modelo PicoPip 379 de LOTEK$^{\circ}$, de $0.6 \mathrm{~g}$ de peso, con una duración de la batería de 40 días aproximadamente, sujeto con un arnés de elástico (Rappole y Tipton, 1991). Previo a su liberación se tomó una muestra de pluma rectriz (R4 derecha) para la determinación del sexo mediante técnicas moleculares.

Durante el periodo comprendido entre el 20 de diciembre de 2013 y 11 de marzo de 2014 ( $n=81$ días), se realizó la localización visual diaria de cada individuo con transmisor con la ayuda del receptor radio ATS (Biotracker') y auriculares (David Clark modelo H10-00-4), confirmando su estatus (viva o muerta) y registrando las coordenadas de su ubicación con la ayuda de un navegador GPS marca Garmin modelo Vista.

Para la estimación del tamaño del ámbito hogareño se utilizaron un mínimo de 20 localizaciones por ave (Farías et al., 2006). El tamaño del ámbito hogareño se estimó con el método de kernel fijo (K) del 95\% con validación cruzada de mínimos cuadrados (Seaman et al., 1999; Powell, 2000). Se utilizó el programa $R$ con las extensiones adehabitatHR y KernSmooth.

Para cada individuo se elaboró un mapa de movilidad geoespacial durante el periodo en que estuvo activo en el área de estudio con la ayuda del software $R^{\circ}$ (R Development Core Team 2015). A partir de este análisis se calcularon las variables distancia promedio (DP) y distancia total recorrida (DT), distancia del punto inicial al final (DIF) y distancia diaria recorrida (DDR) utilizando la distancia euclidiana entre dos puntos de coordenadas cartesianas $\left(P_{1}=\left(X_{1}, Y_{1}\right)\right.$ y $\left.P_{2}=\left(X_{2}, Y_{2}\right)\right)$ (Cuadro 1 y 2).

$$
d_{E}\left(P_{1}, P_{2}\right)=\sqrt{\left(x_{2}-x_{1}\right)^{2}+\left(y_{2}-y_{1}\right)^{2}}
$$

La estimación de la cobertura de suelo y altura de vegetación se realizó usando la metodología descrita por Macías-Duarte y colaboradores (2018), en una área circular de $5 \mathrm{~m}$ de radio teniendo como punto central la georeferencia de la localización de las aves $(n=1404)$, para las variables de cobertura de pasto (\%), cobertura de arbusto (\%), cobertura de hierba (\%), suelo desnudo (\%) y otra cobertura (\%), como excremento animal, vegetación muerta y se medió la altura de pasto $(\mathrm{cm})$, altura de hierba $(\mathrm{cm})$ y altura de arbusto $(\mathrm{cm})$ con el bastón de Robel de $1 \mathrm{~m}$ marcado cada $2 \mathrm{~cm}$ (Robel et al., 1970).

Se realizaron análisis de varianza (ANOVA) sobre las variables de movimiento y ámbito hogareño previamente descritas. Para mejorar su ajuste se omitieron valores atípicos extremos del análisis. Se probó la normalidad de los residuales mediante la prueba de Shapiro-Wilk $(p=0.59)$ y la homogeneidad de las varianzas de los grupos con la prueba de Levene $(p=0.61)$. Las variables de cobertura de suelo y estructura de la vegetación se analizaron mediante análisis de varianza de una vía de Kruskal-Walis $(Z \geq 1.96)$ con prueba de comparación de medianas de Bonferroni. El análisis de los datos estadísticos se realizó mediante el software PAST (Hammer et al., 2001). 


\section{RESULTADOS}

Durante el invierno 2013-2014 se registraron 1,404 puntos de detección de 33 aves monitoreadas durante tres meses: 20 individuos de Centronyx bairdii y 13 de Ammodramus savannarum, con 849 y 555 ubicaciones, respectivamente. El tamaño promedio del ámbito hogareño invernal estimado para BAIS es de 4.82 ha (IC $95 \%= \pm 1.86$ ) y GRSP de 4.38 ha (IC $95 \%= \pm 2.35$; Tabla 1). Las diferencias del tamaño del ámbito hogareño entre las dos especies de gorriones no fueron estadísticamente significativas (Kruskal-Wallis, $\mathrm{Z} \leq$ 1.96). El promedio de DDR (distancia diaria recorrida) (m) fue mayor y estadísticamente diferente en BAIS que en GRSP ( $p$ =0.02), (Tabla 2 y Figura 2), lo que identifica a C. bairdii como una especie más móvil en comparación con $A$. savannarum, sin embargo eso no influye en el tamaño del ámbito hogareño.

\section{Análisis de cobertura de suelo y altura de vegetación}

Se registraron 1,404 detecciones de aves, donde se realizaron igual número de estimaciones de cobertura de suelo y altura de vegetación, para C. bairdii $(n=849)$ y para $A$. savannarum $(\mathrm{n}=555)$.

Los resultados muestran que las variables de cobertura de suelo y vegetación para cada especie no influyeron sobre el tamaño del ámbito hogareño invernal que fue estadísticamente similar entre ellas.

BAIS prefiere sitios con mayor cobertura (\%) que altura de pasto $(\mathrm{cm})$ y de mayor altura de hierba $(\mathrm{cm})$ que GRSP que prefiere lugares con mayor altura de pasto y arbusto, tal y como se observa en la tabla 3.

Tabla 1. Ámbito hogareño invernal (ha), desviación estándar (DE), intervalo de confianza (IC) y numerode individuos ( $n$ ) de Centronyx bairdii (BAIS) y Ammodramus savannarum (GRSP) en Cuchillas de la Zarca, Invierno 20132014.

Table 1. Winter home range (ha), standard deviation (SD), confidence interval (Cl) and number of individuals ( $\mathrm{n}$ ) of Centronyx bairdii (BAIS) and Ammodramus savannarum (GRSP) in Cuchillas de la Zarca, Winter 2013-2014.

\begin{tabular}{ccccc}
\hline Especie & Área (ha) & DE & IC 95\% & $\boldsymbol{n}$ \\
\hline BAIS & $4.82^{\mathrm{a}}$ & 4.24 & \pm 1.86 & 20 \\
GRSP & $4.38^{\mathrm{a}}$ & 4.33 & \pm 2.35 & 13 \\
\hline
\end{tabular}

Literales distintas entre columnas muestran diferencia estadística (KruskalWallis $Z \leq 1.96$ ).

Tabla 2. Estimación media, desviación estándar (DE) y coeficiente de variación (CV) de la distancia total recorrida (DT), distancia inicial y final (DIF) y distancia diaria recorrida (DDR) de Centronyx bairdii y Ammodramus savannarum, durante la temporada invernal 2013-2014 en APCP Cuchillas de la Zarca.

Table 2. Average estimate, standard deviation (SD) and coefficient of variation (CV) of the total distance (DT), initial and final distance (DIF) and daily distance (DDR) of Centronyx bairdii and Ammodramus savannarum, during the winter season 2013 -2014 in GPCA Cuchillas de la Zarca.

\begin{tabular}{lccccccccc}
\hline & \multicolumn{3}{c}{ DT $(\mathbf{k m})$} & \multicolumn{3}{c}{ DIF (m) } & \multicolumn{3}{c}{ DDR (m) } \\
\cline { 2 - 10 } & $\boldsymbol{\mu}$ & DE & CV & $\boldsymbol{\mu}$ & DE & CV & $\boldsymbol{\mu}$ & DE & CV \\
\hline BAIS & 3.37 & 2.38 & 0.70 & 67.49 & 653.87 & 9.68 & 203.13 & 187.07 & 0.92 \\
GRSP & 3.17 & 1.87 & 0.59 & 251.45 & 294.95 & 1.17 & 134.34 & 106.38 & 0.79 \\
\hline
\end{tabular}
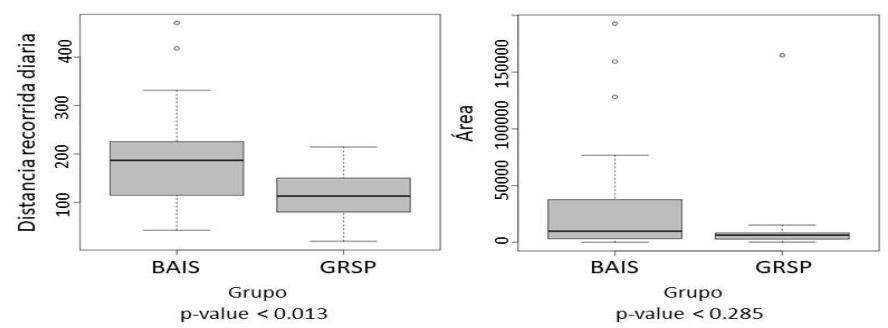

Figura 2. Comparación entre Centronyx bairdii (BAIS) y Ammodramus savannarum (GRSP) para distancia diaria recorrida y tamaño de área invernal, periodo 2013-2014, en del Área Prioritaria para la Conservación de Pastizales (APCP) Cuchillas de la Zarca, Durango, México.

Figure 2. Daily distance traveled and winter area size comparison between Centronyx bairdii (BAIS) and Ammodramus savannarum (GRSP), period 20132014, in the Grasslands Priority Conservation Area (GPCA) Cuchillas de la Zarca, Durango, Mexico.

Table 3. Home range, soil cover and winter vegetation structure variables of Centronyx bairdii and Ammodramus savannarum, in the GPCA Cuchillas de la Zarca.

\begin{tabular}{lcc}
\hline Variable & BAIS & GRSP \\
\hline Ámbito hogareño (ha) & $4.82 \mathrm{a}$ & $4.38 \mathrm{a}$ \\
\hline Altura de pasto (cm) & $31.99 \pm 0.4 \mathrm{~b}$ & $36.06 \pm 0.6 \mathrm{a}$ \\
\hline Cobertura de pasto (\%) & $66.76 \pm 0.6 \mathrm{a}$ & $64.86 \pm 0.8 \mathrm{~b}$ \\
\hline Altura de arbusto (cm) & $21.46 \pm 1.9 \mathrm{~b}$ & $43.15 \pm 1.4 \mathrm{a}$ \\
\hline Cobertura de arbusto (\%) & $1.12 \pm 0.1 \mathrm{~b}$ & $2.86 \pm 0.2 \mathrm{a}$ \\
\hline Altura de hierba (cm) & $40.80 \pm 0.7 \mathrm{a}$ & $32.69 \pm 0.9 \mathrm{~b}$ \\
\hline Cobertura de hierba (\%) & $9.66 \pm 0.3 \mathrm{a}$ & $9.06 \pm 0.4 \mathrm{a}$ \\
\hline Suelo desnudo (\%) & $14.21 \pm 0.6 \mathrm{a}$ & $14.26 \pm 0.7 \mathrm{a}$ \\
\hline Otra cobertura (\%) & $8.22 \pm 0.3 \mathrm{a}$ & $8.93 \pm 0.4 \mathrm{a}$ \\
\hline
\end{tabular}

Literales distintas entre columnas son estadísticamente diferentes $(Z \geq$ 1.96), Centronyx bairdii (BAIS), Ammodramus savannarum (GRSP).

\section{DISCUSIÓN}

Hasta hace algunos años y como resultado de la escasa investigación al respecto, se pensó que las aves de pastizal usan hábitats invernales similares a los utilizados en la época de cría (Wiggins et al., 2006), con base a estudios realizados en su mayoría durante la época reproductiva, donde Davis (2004) encontró en Saskatchewan, Canadá, que Centronyx bairdii, requiere al menos 25 ha de espacio o territorio para reproducción, superficie menor al reporte de Wiggins et al., (2006) donde estiman rangos entre 0.4 - 1.5 ha durante la misma condición reproductiva. Al respecto, en un estudio reciente de sobrevivencia invernal realizado durante tres temporadas de invierno, incluyendo el de este estudio (2013 - 2014) en Janos, Chihuahua, Strasser y Panjabi (2016) reportaron que el ámbito hogareño promedio para $C$. bairdii, fue de 7.18 ha $(n=50)$ usando el método de Kernel fijo (Seeman et al., 1999), área mayor que la reportada en este estudio (4.82 ha, $\mathrm{n}=20$ ), sin embargo los valores estimados para características de cobertura de suelo y vegetación que usó la especie, son distintas entre sitios, Janos, Chihuahua (JANO) y Cuchillas de la Zarca, Durango (CUZA), destacando las variables suelo desnudo con $48.98 \%$ y $14.21 \%$, cobertura 
de pasto de $39.20 \%$ y $66.76 \%$ y altura de pasto de $19.83 \mathrm{~cm}$ y $31.99 \mathrm{~cm}$ en promedio respectivamente $(n=1541, n=849)$. En ese sentido, Macías-Duarte y colaboradores (2017) encontraron para Centronyx bairdii que los valores de cobertura de suelo y estructura de vegetación invernal son similares al reporte de Strasser y Panjabi (2016) en Janos, Chih., pero distintos al reporte de Martínez - Guerrero y colaboradores (2011) para Cuchillas de la Zarca, quienes estimaron valores similares en cobertura de pasto, altura de pasto, cobertura de hierba, suelo desnudo, cobertura de arbusto y otra cobertura que las encontradas durante 2013 - 2014 en el mismo sitio.

Las condiciones entre sitios son distintas, lo que provoca en muchas especies de aves migratorias realizar movimientos estacionales diferentes (Marra, 2011), lo que implica tratar de integrar información no solo de distintos sitios durante una estación, sino también de la época reproductiva en los sitios de crianza bajo el enfoque de modelos de ciclo anual para entender mejor esos cambios, sobretodo en especies con problemas de declinación poblacional (Hostetler et al., 2015), como las del presente estudio.

Para el caso de Ammodramus savannarum, Balent y Norman (2003) estimaron en el suroeste de los Estados Unidos que usa parches de pastizal de 13.2 ha en época reproductiva, en contraste con lo observado por Strasser y Panjabi (2016) en el estudio arriba mencionado, donde reportan para esta especie 4.01 ha $(n=96)$, que concuerda con el reporte del presente estudio ( $4.38 \mathrm{ha}, \mathrm{n}=13$ ).

Las características de la cobertura de suelo y estructura de la vegetación, también difiere entre sitios usados por $A$. savannarum en regiones distintas del desierto chihuahuense, donde destaca la alta tolerancia en JANO (Strasser y Panjabi, 2016) para suelo desnudo $(66.16 \%, n=1497)$, mientras que en CUZA fue mucho menor $(14.26 \%, \mathrm{n}=555)$, cobertura $y$ altura de pasto de $27.80 \%$ y $18.69 \mathrm{~cm}$, mientras que en $\mathrm{Cu}$ chillas de la Zarca fue de $64.86 \%$ y $36.06 \mathrm{~cm}$. Los resultados estimados de movilidad para ambas especies son novedosos y representan una aportación valiosa del presente estudio, que aproximan a entender de qué manera usan el territorio invernal.

Algunos elementos y variables no considerados durante este estudio que pueden ayudar a explicar los resultados encontrados hacen referencia a la competencia homoespecifica y heteroespecifica, al respecto, Pulliam (1985) sugiere la competencia por recursos alimenticios en gorriones de pastizal cuando estos son escasos en años extraordinarios o secos, a su vez, en invierno las aves son exclusivamente granívoras y la producción de semilla del pasto como fuente potencial de alimento (Titulaer et al., 2017) derivado de un verano lluvioso, puede influir también en la estructura de la vegetación (Bertellier y Carrera, 2015), generando fuertes concentraciones de aves de distintas especies que compiten por recursos (Panjabi et al., 2013). Un estudio realizado por Macías-Duarte y colaboradores (2018) en las distintas áreas prioritarias para la conservación de pastizales del desierto chihuahuense entre 2001 y 2007, la densidad promedio de BAIS (7.20 aves $\mathrm{km}^{2}$ ) y GRSP (41.41 aves $\mathrm{km}^{2}$ ) está influenciada por las precipitaciones en verano donde se pudo observar que las densidades son menores a medida que la vegetación es más densa, porque disminuye a su vez la capacidad de detección de ambas especies de aves de pastizal. Este efecto climático, se observa también en el gorrión cola blanca (Poocetes gramineus) donde se encontraron diferencias en el tamaño del territorio para inviernos consecutivos en Janos, Chihuahua, donde Macías-Duarte y Panjabi (2013b) reportan el tamaño del territorio de esta especie en 2009 de $108.46 \pm 36.43$ ha y en 2010 de $30.91 \pm$ 4.74 ha. Otro aspecto importante que puede determinar la variación del tamaño del territorio es el tamaño y forma del pico de ambas especies de estudio, relacionado a la cantidad y tipo de semillas disponibles como alimento, que los capacita para consumir cierto tipo de semillas, influenciado por el tamaño por lo que prefieren aquellas que pertenecen al género Bouteloua y a la familia Paniconidae (Titulaer, 2015), que son muy abundantes y dominantes en el área de estudio.

El comportamiento del movimiento de un organismo está dado por la escala que percibe y que responde a la subdivisión espacial de su entorno (Wiens, 1976; Levin, 1992). El movimiento también es un elemento fundamental del proceso de desarrollo de la población, el flujo genético, la selección del hábitat y el comportamiento del forrajeo y forraje, es un paso importante hacia la comprensión de la dinámica de un organismo. Algunos autores (Dytham, 1994; Tilman et al., 1994; Moilanen y Hanski, 1995) han demostrado que especies sedentarias como $A$. savannarum y $C$. bairdii, se han visto más seriamente afectadas por la fragmentación de los pastizales (De Leon et al., 2014; Pool et al., 2014) que las especies más móviles en sus hábitats invernales o no reproductivos. El tamaño de parche de pastizal que contenga suficiente cobertura y estructura de vegetación además de disponibilidad de alimento (semillas) será el adecuado para que las aves de pastizal migratorias encuentren los recursos para sobrevivir durante el invierno (Brown y Sherry, 2006), sin embargo, la dispersión de estos sitios en el paisaje, el comportamiento sedentario de algunas especies y la inexperiencia de aves jóvenes, produce individuos flotantes (aquellos que no seleccionan un territorio determinado), que compiten por recursos con individuos conespecíficos que por condición de edad y sexo seleccionan los mejores sitios (Brown y Long, 2007), fenómeno que se observa con más frecuencia en especies monomórficas como las del presente estudio (Koronkiewicz et al, 2006).

Una posible explicación para las diferencias en los patrones de movimiento entre los individuos que están presentes en las áreas de invierno, son que algunas tienen rangos de hogar fijos y otros que se mueven de manera nómada. Esta diferencia se puede tomar como consecuencia del comportamiento que ambas especies presentan en los territorios de invernada donde GRSP tiene un comportamiento más sedentario (Gordon, 2000a) y patrones de movimientos más limitados que BAIS (Brown, 1989; Brown y Zeng, 1989). Sin embargo, la estructuración competitiva entre especies no debe ser descartada por completo. La competencia no nece- 
sita ser intensa o constante para efectuar una distribución de las aves dentro del espacio óptimo para la invernada (Connell, 1980). Como Pulliam (1985) menciona, que la distribución y la competencia de los recursos disponibles en las áreas de invernada (Arizona, E.U.A) de los gorriones son importantes solo en años en los cuales la producción de semilla ha sido extremadamente baja y la competencia de la semilla puede ser especialmente intensa (Marone et al., 1992; MéndezGonzalez, 2010). Por consecuencia, esta competencia se ve reflejada en la amplitud del ámbito hogareño, la sobre carga de aves en las áreas de internación, además, incluso si los depredadores limitan a las poblaciones de gorriones por debajo de su capacidad para saturar los recursos alimentarios, puede ser necesaria la partición de nichos para la coexistencia (Holt, 1977).

Esto es coherente con muchos sistemas territoriales por los individuos "flotantes" que representan un exceso de individuos en relación al número de territorios disponibles y ocupados por individuos de mejor condición corporal (Shutler y Weatherhead 1994; Stutchbury, 1994; Westcott y Smith 1994).

\section{CONCLUSIONES}

Se logró estimar el ámbito hogareño invernal de $A$. savannarum y C. bairdii, dos especies gorriones focales de pastizal, ambas estimaciones fueron menores a las reportadas en tierras reproductivas y difieren también entre las reportadas en otras regiones del desierto chihuahuense. La similitud de los territorios entre especies sugiere que la disponibilidad de los recursos durante el invierno de estudio fue suficiente para que las aves no usaran territorios más grandes. Las características de la cobertura de suelo y estructura de la vegetación como hábitat de las especies de estudio, no tuvieron influencia en el tamaño del ámbito hogareño, aunque $C$. bairdii prefiere elementos de mayor cobertura de suelo y que A. savannarum prefiere estructura de la vegetación más alta. La distancia diaria recorrida de BAIS demuestra que es una especie que se desplaza más que GRSP y constantemente está buscando áreas de cobertura y alimento, posiblemente ocasionado por la densidad poblacional de cada especie.

Finalmente se reconoce al GPCA de CUZA como un área importante para la invernada de ambas especies, porque existen condiciones de hábitat adecuadas y se sugiere iniciar acciones encaminadas a la conservación del sitio.

\section{REFERENCIAS}

Balent, K.L. y Normant, C.J. 2003. Demographic characteristics of a grasshopper sparrow population in a highly fragmented landscape of western New York State. Journal of Field Ornithology 74: 341-348.

Bertellier, M. y Carrera, A.L. 2015. Aboveground vegetation and perennial grass seed bank in arid rangelands disturbed by grazing. Rangeland ecology y management 68:71-78.

Brown, J. S. 1989. Desert rodent community structure: A test of four mechanisms of coexistence. Ecological Monographs 59: $1-20$.
Brown D.R. y Long J.A. 2007. What is a winter floater? causes, consequences, and implications for habitat selection. Condor 109: 548-565.

Brown D.R y Sherry T.W. 2006. Food supply controls the body condition of a migrant bird wintering in the tropics. Oecologia149: 22-32.

Brown, J.H. y Z. Zeng. 1989. Comparative population ecology of eleven species of rodents in the Chihuahuan Desert. Ecology 70: 1507-1525.

CEC. 2013. Where do grassland birds winter? Density, abundance and distribution of wintering grassland passerines in the Chihuahuan Desert. Montreal, Canada. Commission for Environmental Cooperation.

Connell, J.H. 1980. Diversity and the coevolution of competitors, or the ghost of competition past. Oikos 35: 131-138.

Davis, S.K. 2004. Area sensitivity in grassland passerines: effects of patch size, patch shape, and vegetation structure on bird abundance and occurrence in southern Saskatchewan. Auk 121: 1130-1145.

De León-Mata, D., Pinedo-Álvarez, A. y Martínez, J.H. 2014. Aplicación de sensores remotos en el análisis de la fragmentación del paisaje en Cuchillas de la Zarca, México. Investigaciones Geográficas Boletín. 84: 42-53.

Dytham, C. 1994. Habitat destruction and competitive coexistence: a cellular model. J. Anim. Ecol. 63: 490-491.

Farías, V., Fuller, T.K., Cervantes, F.A. y Lorenzo, C. 2006. Home range and social behavior of the endangered Tehuantepec Jackrabbit (Lepus flavigularis) in Oaxaca, México. Journal of Mammalogy 87:748-756.

Gordon, C.E. 2000a. Fire and cattle grazing on wintering sparrows in Arizona grasslands. Journal of Range Management 53: 384-389.

Gordon, C.E. 2000b. Movement patterns of wintering grassland sparrows in Arizona. The Auk 117: 748-759.

Hammer, O., Harper, D.A.T., y Ryan, P.D. 2001.PAST:Paleontological Statistics Software Package for Education and Data Analysis. Palaeontologia Electronica 4: 9.

Hoekstra, J.M., Boucher, T.M., Ricketts, T.H. y Roberts, C. 2005. Confronting a biome crisis: global disparities of habitat loss and protection Ecology Letters 8: 23-29.

Holt, R.D. 1977. Predation, apparent competition, and the structure of prey communities. Theoretical Population Biology 12: 197-229.

Hostetler, J.A., Sillett, T.S. y Marra, P.P. 2015. Full-annual-cycle population models for migratory birds. The Auk 132: 433449.

Koronkiewicz, T.J., Sogge, M.K. Van Riper, III C. y H. Paxton, E. 2006. Territoriality, site fidelity, and survivorship of Willow Flycatchers in Costa Rica. Condor 108: 558-570.

Levin, S.A. 1992. The Problem of Pattern and Scale in Ecology: The Robert H. MacArthur Award Lecture. Ecology. 73: 19431967.

Macias-Duarte, A. y Panjabi, A.O. 2013. Association of habitat characteristics with winter survival of a declining grassland bird in Chihuahuan desert grassland of Mexico. The Auk 130: 141-149.

Macías-Duarte, A., Panjabi, A.O., Strasser, E.H., Levandoski, G.J., Ruvalcaba-Ortega, I., Doherty Jr. P.F. y Ortega-Rosas, C.I. 2017. Winter survival of declining North American grassland birds is driven by weather and grassland condition in the Chihuahuan Desert. Journal of Field Ornithology 88: 374-386. 
Macías-Duarte, A., Panjabi, A.O., Pool, D.B., Ruvalcaba-Ortega, I. y Levandoski, G.J. 2018. Fall vegetative cover and summer precipitation predict abundance of wintering grassland birds across the Chihuahuan Desert. Journal of Arid Environments 156: 41-49.

Marone, C., Hobbs, B.E. y Ord, A. 1992, Coulomb constitutive laws for friction: Contrasts in frictional behavior for distributed and localized shear: Pure and Applied Geophysics. 139: 195-214.

Méndez-Gonzalez, C. 2010. Influence of seed resources on the diet, seed selection and community dynamics of wintering birds in semi-arid grasslands. Ph.D. dissertation. New Mexico State University, Las Cruces.

Marra, P. 2011. Studying birds in the context of the annual cycle: Carry-over effects and seasonal interactions. The Ecology and Conservation of Migratory Birds. http://www.bou.org. uk/bouproc-net/migratory-birds/marra.pdf

Martínez-Guerrero J., Wehenkel, C., Pereda, M., Panjabi, A., Levandoski, G., Corral, J., Díaz, R. y Hernández, J. 2011. Abundancia y distribución invernal de Ammodramus bairdii, en la región de Cuchillas de la Zarca, México. Huitzil 12: 9-14.

Martínez-Guerrero, J.H²., Wehenkel, Ch., Pereda-Solís M., Panjabi, A., Levandoski, G., Corral-Rivas, J. y Díaz-Moreno, R. 2011. Relación entre la cobertura de suelo y atributos de la vegetación invernal con Ammodramus bairdii, Audubon, 1844, en el Noroeste de México. Agrociencia. 45:443-451.

Moilanen, A., y Hanski, I. 1995. Habitat Destruction and Coexistence of Competitors in a Spatially Realistic Metapopulation Model. Journal of Animal Ecology 64: 141144.

North American Bird Conservation Initiative, U.S. Committee (NABCl). 2016. The State of the Birds 2016 Report. U.S. Department of Interior, Washington, D.C

http://www.stateofthebirds.org/2016/resources/speciesassessments/

Panjabi, A., G. Levandoski y R. Sparks. 2007. Wintering Bird Inventory and Monitoring in Priority Conservation Areas in Chihuahuan Desert Grasslands in Mexico: 2007 pilot results. Rocky Mountain Bird Observatory, Brighton, CO. Final Technical report I-MXPLAT-TNC07-02.

Panjabi, A. y Beyer, L. 2010. Desert Grassland Bird Conservation: Is low winter survival driving population declines? Report Phase I. Brighton, CO Rocky Mountain Bird Observatory.

Panjabi, A.O., Macías-Duarte, A., Ruvalcaba-Ortega, I., Pool, D., Levandoski, G. y González-Rojas, J. I. 2013. Where do grassland birds winter? Density, abundance and distribution of wintering grassland passerines in the Chihuahuan Desert. Montreal, Canada. Commission for Environmental Cooperation.

Partners in Flight (PIF). 2016. Landbird Conservation Plan. Revision for Canada and continental United States. In: http:// www.partnersinflight.org/wp-content/uploads/2016/08/ pif-continental-plan-final-spread-single.pdf

Pyle, P. 1997. Identification Guide to North American Birds. Part 1. Slate Creek Press, Bolinas, CA.

Pulliam, H.R. 1985. Foraging efficiency, resource partitioning, and the coexistence of sparrow species. Ecology 66: 18291836.

Pool, B.D., Panjabi, A., Macias-Duarte, A. y Soljhem, D. 2014. Rapid Expansion of Croplands in Chihuahua, Mexico Threatens
Declining North American Grassland Bird Species. Biological Conservation. 170: 274-281.

Powell, R.A. 2000. Animal home ranges and territories and home range estimators. En: Research techniques in animal ecology: controversies and consequences. L. Boitani y T. K. Fuller (ed.). 65-110 pp. Columbia University Press, New York.

R Development Core Team. 2015. R: A Language and Environment for Statistical Computing. R Foundation for Statistical Computing, Vienna. Disponible en cran.r-project. org/doc/manuals/refman.pdf.

Rappole, J.H. y Tipton A.R. 1991.New Harnes Design for Attachment of Radio Transmitters to Small Passerines. Journal of Field Ornithology 62: 335-337.

Robel, R.J., Briggs, J.N., Dayton, A.D. y Hulbert, L.C. 1970. Relationships between visual obstruction measurements and weight of grassland vegetation. Journal of Range Management 23: 295-297.

Ruth, J.M., Stanley, T.R., y Gordon, C.E. 2014. Associations of wintering birds with habitat in semidesert and plains grasslands in Arizona. The Southwestern Naturalist. 52: 199211.

Sauer, J. R., Niven, D.K., Hines, J.E., Ziolkowski, Jr D.J., Pardieck, K.L., Fallon, J.E. y Link, W. A. 2017. The North American Breeding Bird Survey, Results and Analysis 1966 - 2015. Version 2.07.2017 USGS Patuxent Wildlife Research Center, Laurel, MD.

Seaman, D., E., Millspaugh, J. J., Kernohan, B. J., Brundige, G. C., Raedeke, K. J. y Gitzen, R. A. 1999. Effects of sample size on kernel home range estimates. Journal of Wildlife Management 63: 739-747.

Shutler, D. y Weatherhead, P.J. 1994. Movement patterns and territory acquisition by male red-winged blackbirds. Can J Zool 72:712-720.

Somershoe, S.G., Brown C.R.D. y Poole, R.T. 2009. Winter site fidelity and over-winter site persistence of passerines in Florida. Wilson Journal of Ornitholgy 121: 119-125.

Sauer, J. R., D. K. Niven, J. E. Hines, D. J. Ziolkowski, Jr, K. L. Pardieck, J. E. Fallon, y W. A. Link. 2017. The North American Breeding Bird Survey, Results and Analysis 1966 - 2015. Version 2.07.2017 USGS Patuxent Wildlife Research Center, Laurel, MD.

Seaman, D.E, Millspaugh, J.J., Kernohan, B.J., Brundige, G.C., K. J. Raedeke, y Gitzen, R.A.1999. Effects of Sample Size on Kernel Home Range Estimates. Journal of Wildlife Management 63: 739-747.

Strasser, E.H. y Panjabi, A.O. 2016. Identifying limiting factors for grassland birds wintering in the Chihuahuan Desert. Bird Conservancy of the Rockies, Brighton, CO, Final report submitted to U.S. Forest Service International Program.

Stutchbury, B.J. 1994. Competition for winter territories in a Neotropical migrant: the role of age, sex and color. Auk 111: 63-69.

Tilman, D., May, R.M., Lehman, C.L., Nowak, M.A. 1994. Habitat destruction and the extinction debt. Nature 371:65-66.

Titulaer, M. 2015. Selección de semillas y dieta invernal de aves de pastizal en el norte de México. Ph. D. dissertation. Universidad Autónoma de Chihuahua, Facultad de Zootecnia y Ecología. Chihuahua, México.

Titulaer, M., Melgoza-Castillo, A., Panjabi, A.O., Sánchez-Flores, A., Martínez-Guerrero, J.H., Macías-Duarte, A. y FernándezFernández, J. A. 2017. Molecular analysis of stomach 
contents reveals important grass seeds in the winter diet of two declining grassland bird species. PLOS ONE: 12: e0189695. https://doi.org/10.1371/journal.pone.0189695

Vickery, P. y Herkert, J. 2001. Recents advances in grassland bird research: Where do we go from here? The Auk 118: 11-15.

Westcot, D.A., y Smith, J.N.M. 1994. Behavior and social organization during the breeding season in Mionectes oleagineus, a lekking flycatcher. Condor 96: 672-683.
Wiens, J.A. 1976. Population responses to patchy environment s. Annual Review of Ecology and Systematics 7:81-21.

Wiggins, D.A. 2006. Baird's Sparrow (Centronyx bairdii): a technical conservation assessment. USDA Forest Service, Rocky Mountain Region. Web site: http://www.fs.fed.us/ r2/projects/scp/assessments/bairdssparrow.pdf [accessed October 2016]. 